\title{
Promiscuous Geometries
}

\section{XHULIO BINJAKU}

Massachusetts Institute of Technology

Promiscuous Geometries is a set of ornamental objects created from shadows. This project looks to shadows as a medium for design. Shadows are a resultant of light obscured from a surface-shadows come aftergeometry. However, this project reverses that order and starts with shadows to make geometry. The resultant geometry is not a pre-rationalized form but found through a mixing of shadows-a shadowplay. The resulting geometry is surprising and ambiguous, we call it promiscuous, however closely tied to an understanding of geometry. While shadows are clearly important to architecture-no building, or photo of building, would be complete without them-they have typically been used in characteristically experiential terms, as exemplified in Jun'ichirō Tanizaki 1933 essay In Praise of Shadows, while the shadows geometric capabilities have been underused. Promiscuous Geometries begins with shadows and uses anamorphic projection and Boolean operations to produce a set of 3D geometries.

To create a Promiscuous Geometry, begin by drawing any two shadows which are closed. Now loft theses shadows onto two points in space opposite from one another. When a closed shape is lofted to a point, a conic surface results, similar to the art pieces Solid Light Worksby Anthony McCall. When the shadows are lofted to opposite points, their resulting shadow cones intersect. Using a Boolean intersection between the two shadow cones results in a closed geometry with each of its surfaces being conic. Since the resulting geometry is conic, its surfaces are developable, and the geometry can be unrolled flat and reproduced again from flat sheets. We laser cut the panels and assembled them together to form the 3D geometry, attached a string to hang and filled the geometry with white plaster. Once set, the geometry contains the original shadow data which can be shown when a point light, like a phone flashlight, shines onto the geometry. When two point lights are on the geometry, both shadows can be seen simultaneously. Therefore, a reciprocity is built between the Promiscuous Geometry and the shadows from which it was drawn. This experiment can be repeated with various shadow shapes.

While this project shared affinities with post-digital project, namely its witty, vague and primitive forms, it not regressive. And thought the project proposes a formula of combining shadows that can result in endless generative forms, it is not techno-optimists. Like the shadow art of Tim Noble and Sue Webster, from which this project takes inspiration,
ANNE GRAZIANO

Massachusetts Institute of Technology

the project is a resultant of a clear understanding of geometry that speaks to the longer history of shadows as a useful design tool. With shadows architects can producegeometry, instead of simply consumeit as Robin Evans stated. 


\section{PROMISCUOUS GEOMETRIES A set of geometries that are drawn from shadows.}

This project uses anamorphic projection and boolean operations to create a set of geometries that are drawn from shadows. Two shadows are drawn on a flat surface, like a table, the resulting shapes are lofted to a set point, producing two conic geometries. The two shadow cones are then intersected, resulting in a 3D geometry that is at once ambiguous, we call it "promiscuous," but also contains the original shadow data. When the geometry is lit from pointlights, the original shadows once again becomes visible.
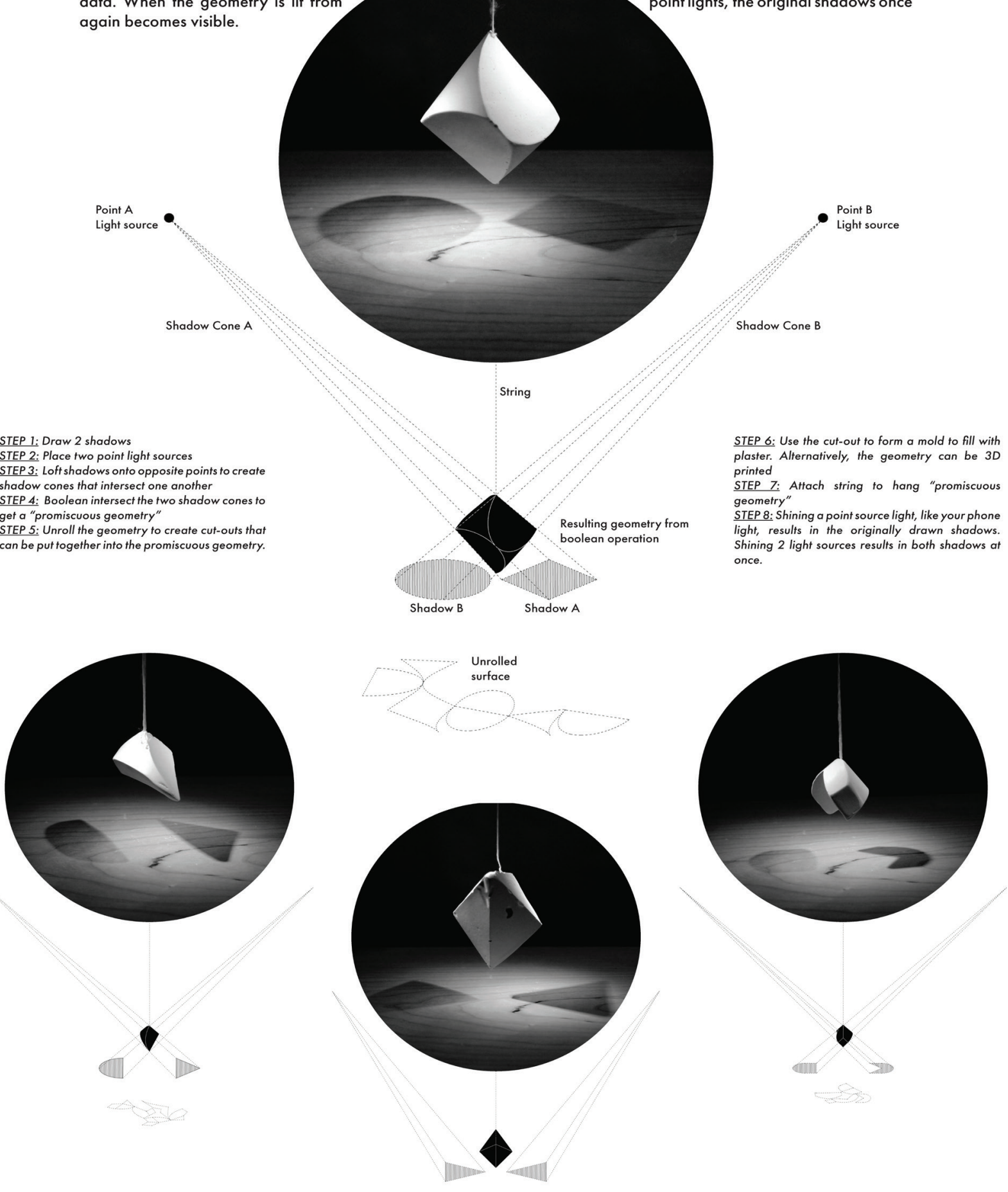
STEP 6: Use the cut-out to form a mold to fill with printed

STEP 7: Attach string to hang "promiscuous geometry"

STEP 8: Shining a point source light, like your phone light, results in the originally drawn shadows. tight sources results in both shadows at once.

Point A

Shadow Cone A Shadow B 